\title{
Are lizards capable of inhibitory control? Performance on a semi-transparent version of the cylinder task in five species of Australian skinks
}

\author{
Birgit Szabo $^{1,2}$ (D) Sebastian Hoefer ${ }^{1,3}$ (D) Martin J. Whiting ${ }^{1}$ (D)
}

Received: 4 August 2020 / Revised: 13 August 2020 / Accepted: 20 August 2020 / Published online: 7 September 2020

(C) The Author(s) 2020

\begin{abstract}
Inhibitory control, the inhibition of prepotent actions, is essential for higher-order cognitive processes such as planning, reasoning, and self-regulation. Individuals and species differ in inhibitory control. Identifying what influences inhibitory control ability within and between species is key to understanding how it evolved. We compared performance in the cylinder task across five lizard species: tree skinks (Egernia striolata), gidgee skinks (Egernia stokesii), eastern blue-tongue skinks (Tiliqua s. scincoides), sleepy lizards (Tiliqua $r$. asper), and eastern water skinks (Eulamprus quoyii). In our task, animals had to inhibit the prepotent motor response of directly approaching a reward placed within a semi-transparent mesh cylinder and instead reach in through the side openings. Additionally, in three lizard species, we compared performance in the cylinder task to reversal learning to determine the task specificity of inhibitory ability. Within species, neither sex, origin, body condition, neophobia, nor pre-experience with other cognitive tests affected individual performance. Species differed in motor response inhibition: Blue-tongue skinks made fewer contacts with the semi-transparent cylinder wall than all other species. Blue-tongue skinks also had lower body condition than the other species which suggest motivation as the underlying cause for species differences in task performance. Moreover, we found no correlation between inhibitory ability across different experiments. This is the first study comparing cylinder task performance among lizard species. Given that inhibitory control is probably widespread in lizards, motor response inhibition as exercised in the cylinder task appears to have a long evolutionary history and is likely fundamental to survival and fitness.
\end{abstract}

\section{Significance}

The study of lizard cognition is receiving increasing attention. Lizards are a diverse group with a wide range of ecological attributes and represent a model system through which we can test a wide range of hypotheses relating to cognitive evolution. Furthermore, considering their evolutionary history, studying non-avian reptile cognition can help understand the evolution of different cognitive abilities including inhibitory control. Here, we provide a comparison of inhibitory control ability in five lizard species. Consequently, we are able to, firstly, validate a method (the cylinder task) initially developed for the use in mammals and birds, for use in lizards, and secondly, collect valuable data on inhibitory control in a poorly studied group with respect to cognitive ability. Our study suggests noncognitive factors as a major influence on cylinder task performance, which is in agreement with previous studies of other vertebrates.

Communicated by T. Madsen

Electronic supplementary material The online version of this article (https://doi.org/10.1007/s00265-020-02897-y) contains supplementary material, which is available to authorized users.

Birgit Szabo

birgit.szabo@gmx.at

1 Department of Biological Sciences, Macquarie University, Sydney, NSW 2109, Australia

2 Division of Behavioural Ecology, Institute of Ecology and Evolution, University of Bern, Bern, Switzerland

3 Department of Biology, Freie Universität, Berlin, Germany
Keywords Cognition - Executive functions - Response inhibition $\cdot$ Non-avian reptile $\cdot$ Squamate reptile

\section{Introduction}

To maximize gain in a given situation, animals often need to exercise inhibitory control over their behavior (Diamond 2013). For example, failure to inhibit premature strikes towards potential prey or relying on irrelevant information during environmental changes could have negative consequences for fitness. Moreover, inhibitory control is one of several basic processes that are part of executive functions (also cognitive 
control; Diamond 2013), necessary for executing higher-level cognitive skills such as planning, reasoning, and selfregulation (Diamond 2013). Inhibiting harmful or disadvantageous behavior, especially in the presence of an external pull, could be an important skill for survival.

Frequently, inhibitory control is tested using reversal learning tasks, A-not-B tests, or detour tasks. During reversal learning tasks, in which a previously established stimulus-reward relationship changes, animals need to inhibit responding to the formerly rewarded stimulus (now unrewarded; e.g., Boogert et al. 2011; Anderson et al. 2016; Brucks et al. 2018; Ducatez et al. 2019) and form a new association with the previously unrewarded stimulus and the reward (Dias et al. 1996). In the A-not-B test, in which a reward is first hidden in location $\mathrm{A}$ and then moved to location B in full view of the subject for a single test trial, animals need to inhibit prepotent responses towards location A (Bray et al. 2014; Vernouillet et al. 2018). Finally, detour tasks involve retrieving a reward from behind a barrier which can be opaque, semi-transparent, or fully transparent. A clear visibility of the reward exerts a stronger urge for a direct reach (Diamond 1981, 1990; Santos et al. 1999; Kabadayi et al. 2017a), and animals need to inhibit this direct response and make a detour around the barrier to reach behind it and obtain the reward (e.g., Diamond 1990; Regolin et al. 1994; Bojczyk and Corbetta 2004; Shaw 2017; Ducatez et al. 2019). One commonly used detour task is the "cylinder task" (MacLean et al. 2014), a two-stage response inhibition task, in which animals are first trained to make a detour to the side openings of an opaque cylinder containing a reward which is invisible prior to approach. Thereafter, the opaque cylinder is replaced with a transparent cylinder to test motor response inhibition by quantifying successful detours when a reward is visible (e.g., Boogert et al. 2011; Bray et al. 2014; MacLean et al. 2014; Anderson et al. 2016; Kabadayi et al. 2016, 2017b; Vernouillet et al. 2016, 2018; Lucon-Xiccato et al. 2017; Bobrowicz and Osvath 2018; Isaksson et al. 2018; Szabo et al. 2019b).

A number of non-cognitive factors can affect detour task performance across individuals: the distance to the goal (often a food reward), reward visibility through the barrier, barrier shape, size and material, motivation to reach the goal, neophobia, rearing conditions, and previous experience with transparent or semitransparent objects (for a full review see Kabadayi et al. 2017a). For example, long-tailed macaques (Macaca fascicularis) try to reach more often directly for a food reward behind a Plexiglas screen when the reward is positioned close to the barrier as opposed to further away (Junghans et al. 2016). Similarly, cats (Felis catus) made less contact with the surface of a transparent cylinder when it was larger (and they could fit their head into it not just their paw) and when the reward was placed further away from a barrier (Bobrowicz and Osvath 2018). Mice (Mus musculus) spent more time in front of a transparent barrier and subsequently took longer to escape out of a water pool than when facing a semi-transparent or opaque barrier (Juszczak and
Miller 2016). Pheasant chicks (Phasianus colchicus) performed better on a novel detour task if they had previously experienced testing on a similar task (differently shaped barrier) while neophobia and body condition had no effect on performance (van Horik et al 2018). In North Island robins (Petroica longipes) pre-experience with a motor task had no effect on detour performance, but birds in better body condition solved the detour task in fewer trials (Shaw 2017). Finally, males performed better on a detour task compared to females in the guppy (Poecilia reticulata; Lucon-Xiccato et al. 2019). Besides these noncognitive factors, individuals might learn how to reach a reward behind a barrier across multiple trials (e.g., Boogert et al. 2011; Vernouillet et al. 2016).

The proficiency with which inhibitory control is executed also differs among species (e.g., MacLean et al. 2014; Kabadayi et al. 2016, 2017b; Rudolph and Fichtel 2017; Brucks et al. 2018). In the case of cylinder tasks, the best scores were achieved by great apes (MacLean et al. 2014), corvids (Kabadayi et al. 2016), and carnivores such as dingos, dogs, and cats (MacLean et al. 2014; Bobrowicz and Osvath 2018). Proficiency on the cylinder task was linked to absolute brain size across 36 species. A comparison among non-human primates revealed that dietary breadth (number of food categories in the diet) but not frugivory or average social group size predicted performance (MacLean et al. 2014) while spotted hyenas (Crocuta crocuta) from larger social groups showed better cylinder task performance (Johnson-Ulrich and Holekamp 2020). Inhibitory control is a phylogenetically widespread ability that varies among individuals as well as species indicating that this ability might be selected for in certain circumstances, but why some species possess enhanced inhibitory control is still unclear.

So far, measures of inhibition have failed to correlate across tasks suggesting that these tasks measure different cognitive skills (e.g., Regolin et al. 1994; Boogert et al. 2011; Bray et al. 2014; Anderson et al. 2016; Fagnani et al. 2016; Brucks et al. 2018; Vernouillet et al. 2018; Ducatez et al. 2019; Szabo et al. 2019b). For example, in male song sparrows (Melospiza melodia), cylinder task performance was very weakly and nonsignificantly negatively correlated with reversal learning performance (Boogert et al. 2011; Anderson et al. 2016). The addition of data from females reduced this correlation to zero (Anderson et al. 2016). In water skinks (Eulamprus quoyii), individuals that learnt a color discrimination performed less well on a semitransparent cylinder task and showed a weaker side bias in the discrimination task (Szabo et al. 2019b). Furthermore, measures of response inhibition have also failed to correlate across contexts. For instance, the ability of wolves (Canis lupus) and dogs (Canis familiaris) to retrieve a reward from behind a V-shaped barrier was weakly positively, and non-significantly, correlated with their success rate in retrieving a reward from within a cylinder (Marshall-Pescini et al. 2015).

Here, we report the performance of four related lizard species on a semi-transparent version of the cylinder task. A fifth species, 
the eastern water skink (Eulamprus quiyii), was tested previously (Szabo et al. 2019b), and these data were included in a species comparison of task performance. All species tested in this study are members of the Australian Egernia group, a radiation of scincid lizards comprising seven genera and approximately 50 species (Chapple 2003; Gardner et al. 2008; While et al. 2015). We tested (1) tree skinks (Egernia striolata), a medium-sized, diurnal, omnivorous lizard species inhabiting arboreal and rocky habitats (Cogger 2014). (2) Gidgee skinks (Egernia stokesii), which are also medium-sized lizards (slightly larger and stockier than tree skinks), diurnal, omnivorous lizards that inhabit arid or semi-arid rocky habitats (Duffield and Bull 1998). (3) Sleepy lizards (Tiliqua rugosa asper) occur in arid habitats, are terrestri$\mathrm{al}$, and are relatively large-bodied, diurnal, omnivorous foragers (Bull 1995; Cooper 2000; Bull et al. 2017), and finally, (4) eastern blue-tongue skinks (T. s. scincoides) are relatively large-bodied, diurnal, and omnivorous lizards (Koenig et al. 2001; Cogger 2014) (Online Resource Table S1).

Our aim was to collect data on the inhibitory ability of lizards in the cylinder task to extend our knowledge on inhibitory control as measured by the cylinder task, across species. A number of reptile species have been documented to successfully detour around barriers (e.g., Burghardt 1978; Wilkinson and Huber 2012; Szabo et al. 2019b), and accordingly, we expected all tested species to learn the detour task and exhibit motor response inhibition. We were interested in finding out if sex, neophobia, body condition, origin of animals, and preexperience with non-related cognitive assays affected performance. All of these factors influence performance in different species (van Horik et al. 2018; Shaw 2017; Lucon-Xiccato et al. 2019; for more examples see Kabadayi et al. 2017a) and due to the lack of data on reptiles, we have only limited knowledge about if and how such factors might influence performance in lizards. Similarly, we were interested in whether species differed in their cylinder task performance and if individual inhibitory control performance correlated across different tasks (the cylinder task and discrimination reversal learning). Collectively, our experiments add additional data to the growing body of knowledge on animal inhibitory control on a modified version of the cylinder task using a semi-transparent mesh cylinder in poorly studied vertebrates. Importantly, our study is the first to compare performance in the cylinder task among lizard species and investigates possible factors that might affect performance within and between lizard species.

\section{Methods}

\section{Study animals and husbandry}

We tested four species on the cylinder detour task: tree skinks (E. striolata, $N=12$, all females), gidgee skinks (E. stokesii, $N=19$, undetermined sex), sleepy lizards (T.r. asper, $N=13$,
7 males and 6 females) and eastern blue-tongue skinks ( $T . s$. scincoides, $N=12,7$ males and 5 females) (Online Resource Table S2) and included one additional species, the eastern water skink (Eulamprus quoyii) for which data were available from a previous study (Szabo et al. 2019b) resulting in five species for comparison. All lizards, except for three adult blue-tongue skinks and three gidgee skinks, had previously been tested in a cognitive assay (for details, see Online Resource Table S2) in which they had the opportunity to gain experience with mesh affordances (semi-transparent material). The data collected on these naïve individuals shows no marked difference compared to the experienced lizards (Online Resource Table S2), and the amount of experience (trials in other tasks) did not affect performance (for details see "Results" section below).

All lizards were moved into individual plastic box enclosures before testing. Because all tested species differed substantially in body (snout-vent, SVL) length (range: $E$. striolata $=101-114 \mathrm{~mm} ;$ E. stokesii $=146-202 \mathrm{~mm} ; T$. $r$. asper $=267-334 \mathrm{~mm} ;$ T. s. scinoides $=157-322 \mathrm{~mm} ; E$. quoyii $=100-118 \mathrm{~mm}$; Online Resource Table S2), enclosures and equipment were scaled to body size to ensure that important task parameters (e.g., relative distance to the cylinder) were the same for all species: tree skinks, juvenile bluetongue skinks, and gidgee skinks were kept and tested in small enclosures $(683 \mathrm{~L} \times 447 \mathrm{~W} \times 385 \mathrm{H} \mathrm{mm})$; adult blue-tongue skinks and sleepy lizards were kept and tested in large enclosures $(800 \mathrm{~L} \times 600 \mathrm{~W} \times 450 \mathrm{H} \mathrm{mm})$. Where possible, we determined sex either by confirming the presence of hemipenes or by using morphological measurements (as per Bull and Pamula 1996; Phillips et al. 2016), and we measured SVL $( \pm 1 \mathrm{~mm})$ and weighed $( \pm 1 \mathrm{~g})$ all lizards before the start of testing. Lizards were individually identified by a PIT tag (Passive Integrated Transponder, Biomark, HPT8, $8.4 \mathrm{~mm} \mathrm{~L} \times 1.4 \mathrm{~mm}$ diameter, $33 \pm 5 \mathrm{mg}$ ) or individual markings and body coloration.

The temperature in the experimental room was set at $24{ }^{\circ} \mathrm{C}$ (resulting in a mean $\pm \mathrm{SD}$ of $24 \pm 2{ }^{\circ} \mathrm{C}$, depending on season), and humidity was between 30 and $60 \%$ depending on season and weather. A heat cord was installed underneath each enclosure to elevate the temperature on one side to $32{ }^{\circ} \mathrm{C}\left( \pm 2{ }^{\circ} \mathrm{C} \mathrm{SD}\right)$. This enabled lizards to thermoregulate to optimal body temperature regardless of overall room temperature. We monitored temperature within enclosures using iButtons (Thermochron iButton model DS1921, recording hourly) for the duration of trials. Animals were habituated to a 12-h light cycle. Lizard enclosures were lined with butcher's paper and each enclosure included a refuge, a water bowl, and two wooden ramps (for more details see Szabo et al. 2018, 2019a; Szabo and Whiting 2020; Online Resource). To prevent respiratory infections in sleepy lizards, which are adapted to low humidity, we introduced a water bowl only twice a 
week for $40 \mathrm{~min}$. All other animals had ad libitum access to water.

When not in experiments, all adult lizards were fed three times per week. Tree skinks received half a teaspoon of baby food (Heinz ${ }^{\mathrm{TM}}$, various flavours) twice a week and crickets powdered with vitamins (aristopet Reptivite) and calcium (URS Ultimate Calcium) once per week. Blue-tongue skinks and sleepy lizards were fed an assortment of small cut fruits and vegetables mixed with a teaspoon of dog food (various brands and flavors) and powdered with calcium (once a week). Gidgee skinks were fed fruits and vegetables three times per week supplemented with crickets (powdered with vitamins and calcium) on 1 day and juvenile blue-tongue skinks were fed fruits and vegetables and high-protein cat food (Purina Supercoat ${ }^{\circledR}$ Adult chicken, powdered with vitamins and calcium) 5 days a week to ensure healthy growth. Diet composition fed during the time of captivity was based on natural diet composition. During the time of experimental trials, lizards were given their regular diet only on Fridays after trials had finished.

\section{Setup}

During trials, masking tape secured the butcher's paper substrate to the enclosure floor to prevent lizards from crawling underneath and out of view. Before the start of the first cylinder task trial we removed everything except the water bowl and refuge from the enclosure (returned after an individual had completed the whole experiment). All lizards started from approximately the same starting position at the end of the enclosure opposite to the cylinder (Fig. 1a). Gidgee skinks were larger than tree skinks and juvenile blue-tongue skinks, but no intermediate sized enclosures were available. We ensured that the relative distance to the cylinder was $1.5 \times \mathrm{SVL}$ (same as for the other four species tested) by moving each gidgee skink by hand back so that their body was parallel to the back wall of the enclosure at the start of each trial (Online Resource Fig. S2). During trials, a small quantity of food reward was inserted into the cylinder placed in the middle, equidistant from the side openings and the cylinder walls (Online Resource Fig. S2). The appropriate amount and type of food used as reward for each species was determined during regular husbandry and adjusted to each species dietary requirements and preference to decrease differences in food motivation which can affect task performance (Kabadayi et al. 2017a). Tree skinks and gidgee skinks were tested using baby food $(0.08 \pm 0.01 \mathrm{~g} /$ trial; this was a sufficient quantity to ensure continuing motivation in both species). We used cat food $(0.2 \pm 0.05 \mathrm{~g} /$ trial $)$ for juvenile blue-tongue skinks and dog food ( $3 \pm 0.2 \mathrm{~g} /$ trial) for adult blue-tongue skinks and sleepy lizards.

\section{Procedure}

Lizards were tested in three trials per day, five days a week, resulting in 15 trials per week and each trial lasted $1.5 \mathrm{~h}$. A trial started by moving a lizard backwards to the starting position by hand and placing the refuge over the lizard to hold it in place until the trial started (Fig. 1a). Next, either a food dish (pre-training) or a cylinder (opaque or semi-transparent depending on test phase) were introduced about $10-15 \mathrm{~cm}$ from the back enclosure wall (in front of the water bowl if present; Fig. 1a). To ensure that lizards could not move the cylinders, we secured them to the enclosure floor using Bostik Blu Tack adhesive putty. Thereafter, the refuge was removed and the lizard was allowed to approach the food dish/cylinder. At the end of the trial, the refuge was put back into the enclosure and the dish/cylinder was removed and cleaned (removing any food that was not eaten with a paper towel) for the next trial.

Tree skinks were tested from March to April 2018, bluetongue skinks from April to August 2018, sleepy lizards from April to October 2018, and gidgee skinks from February to April 2019. These time periods coincided with each species' active season (Cogger 2014) except for sleepy lizards; however, sleepy lizards are active outside their main activity season if resources allow (Bull et al. 2017). No behavioral or motivational changes were detected during the course of trials in any species. Setup and procedures closely followed Szabo et al. (2019b).

\section{Habituation}

To reduce stress during trials, all lizards were tested in their home enclosure (Langkilde and Shine 2006) and left undisturbed for a minimum of 1 week ( 1 week for tree skinks as they had been in captivity for more than 1 year; wild-caught individuals were habituated for longer: blue-tongue skinks and sleepy lizards for 2 weeks and gidgee skinks for 4 weeks) prior to the start of testing (discrimination learning or cylinder task, dependent on what was tested first; Online Resource Table S2). During this time, we made sure that animals were feeding consistently and were habituated to captivity. Lizards were considered habituated when stress-induced behavior (immediate fleeing and hiding) in the presence of the experimenters were greatly reduced (less than once every few visits). Species differed in the length of time needed to reach this criterion.

\section{Pre-training}

All lizards that were tested in discrimination learning before being tested on the cylinder task $(N=9$ blue-tongue skinks, $N=13$ sleepy lizards, $N=16$ gidgee skinks; Online Resource Table S2) were pre-trained for the cylinder task 2 days after they had finished testing. Naïve individuals were pre-trained 


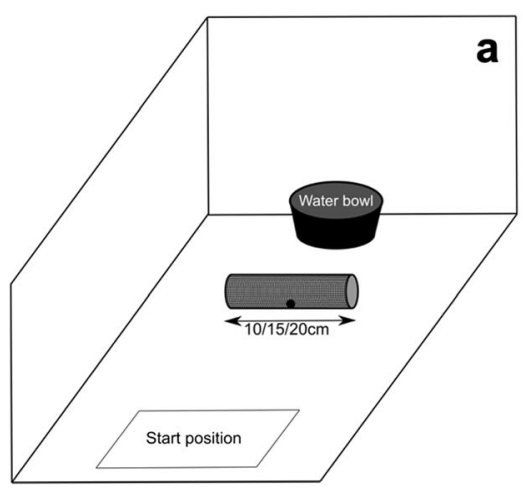

Fig. 1 a An Overview of the setup used for the cylinder task. An opaque or semi-transparent cylinder was attached to the enclosure floor (with Bostik Blu Tack adhesive putty) in front of the water bowl. Due to differences in lizard size, the enclosure and contents were size-adjusted (scaled) to keep non-cognitive factors equal between species (created

immediately after habituation. We pre-trained lizards using food dishes (55-mm-diameter petri dishes with the outside covered in black electric tape for tree skinks and gidgee skinks, or 95-mm-diameter black, plastic food containers with the sides cut down to $20 \mathrm{~mm}$ for blue-tongue skinks and sleepy lizards). One empty dish was placed in front of the water bowl, then, the refuge was removed. In the first five trials, the food was placed inside the dish before the refuge was removed. In the following 10 trials, a piece of food was slowly placed in the food dish (with forceps for dog and cat food or a spoon for baby food) after the refuge was removed and in full view of the subject. This procedure ensured that animals were habituated to the movement of the experimenter's hand inserting food during trials, and thereby reduced stress during food presentation. Furthermore, pre-training was used to determine that lizards were sufficiently food motivated and adequately bold to participate in trials. Criterion for completion of the pre-training was retrieving the reward in at least $75 \%$ of the 15 trials without fleeing during food presentation; $100 \%$ of individuals reached this criterion.

\section{Opaque and semi-transparent cylinder test}

In the opaque cylinder phase, we introduced an opaque cylinder made from PVC and covered in a fine polyester window screen (Cyclone PVC coated Polyester pet mesh screening). The window screen was added to increase similarity between the cylinders used in the different phases. Lizards received a maximum of 30 trials in the opaque cylinder phase and moved on to be tested in the semi-transparent phase if they entered the cylinder at either side without touching its surface in $4 / 5$ consecutive trials (MacLean et al. 2014). Lizards that did not reach this criterion were not tested in the semi-transparent cylinder phase (tree skinks: $N=5$; gidgee skinks: $N=1$;

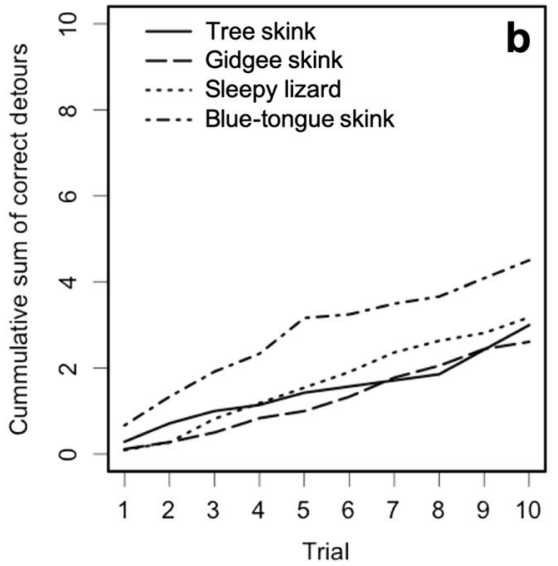

with Inkscape and modified in Microsoft PowerPoint). b The cumulative average group score (successful detours without touching the surface of the cylinder) for each species over the 10 trials given in the semitransparent cylinder phase (created with $\mathrm{R}$ base plot and modified in Microsoft PowerPoint)

sleepy lizards: $N=2$; blue-tongue skinks: $N=0$; water skinks: $N=3$; Table 1). Thereafter, in the semi-transparent cylinder phase, we used a semi-transparent cylinder made from a stiff aluminum insect screen (same grid size as the mesh used in the opaque phase). A mesh cylinder was chosen to facilitate an even food odor diffusion. Lizards were given 10 trials in the semi-transparent cylinder phase (MacLean et al. 2014).

Cylinders were scaled to lizard size because species greatly differed in body length (opening size can affect performance, see Bobrowicz and Osvath 2018). This ensured that all animals could easily pass through the cylinders with their whole body. Tree skinks had the smallest cylinders $(10 \mathrm{~cm}$ long $\times$ $2.9 \mathrm{~cm}$ diameter); juvenile blue-tongue skinks and gidgee skinks had medium-sized cylinders $(15 \mathrm{~cm}$ long $\times 7.5 \mathrm{~cm}$ diameter); and adult blue-tongue skinks and sleepy lizards had the largest cylinders $(20 \mathrm{~cm}$ long $\times 10.3 \mathrm{~cm}$ diameter). The only way to reach the reward in a cylinder was through the designated side openings.

\section{Video scoring}

We recorded all trials using a CCTV system (H.264 Digital Video Recorder, 3-Axis Day and Night Dome Cameras) for later scoring. From the footage, we recorded if a lizard made a correct (1) or incorrect (0) detour response (Bernoulli variable) as well as trial latency (measured from the removal of the refuge until the cylinder was touched or the whole head inserted through one of the side openings of a cylinder) and choice latency (measured from the first forward directed whole body movement until the cylinder was touched or the whole head inserted through one of the side openings of a cylinder). A detour was scored as correct if the lizard entered a cylinder from either side without touching its surface, while an incorrect detour was scored if the lizard touched the outer 
Table 1 Number of individuals that were tested in each phase $(N)$, mean (standard deviation, SD), and range of trials to criterion needed to learn the opaque cylinder test and correct detours to the side of the semitransparent cylinder for each species. Because only those individuals that reached the learning criterion in the opaque cylinder phase were tested in the semi-transparent phase, sample sizes are reduced in the semitransparent test and the number of removed $(N)$ is given

\begin{tabular}{|c|c|c|c|c|c|c|c|}
\hline \multirow[t]{2}{*}{ Species } & \multicolumn{4}{|c|}{ Opaque cylinder phase (trials to criterion) } & \multicolumn{3}{|c|}{ Semi-transparent phase (score out of 10) } \\
\hline & $N$ & Mean (SD) & Range & $N$ removed & $N$ & Mean (SD) & Range \\
\hline Tree skink & 12 & $12.6(6.7)$ & $5-25$ & 5 & 7 & $3(1.6)$ & $1-5$ \\
\hline Gidgee skink & 19 & $14.2(7.3)$ & $5-29$ & 1 & 18 & $2.6(1.2)$ & $1-5$ \\
\hline Sleepy lizard & 13 & $9.1(5.9)$ & $5-26$ & 2 & 11 & $3.2(1.9)$ & $1-7$ \\
\hline Blue-tongue skink & 12 & $8.6(5.1)$ & $5-23$ & 0 & 12 & $4.5(1.4)$ & $2-6$ \\
\hline Water skink & 19 & $13.4(6.9)$ & $5-29$ & 3 & 16 & $4(2.2)$ & $1-8$ \\
\hline
\end{tabular}

surface of the cylinder at any point in the trial before entering through the side openings to consume the reward. To be able to analyze possible effects of neophobia/neophilia on lizard performance, we scored the time a lizard spent within $2 \mathrm{~cm}$ of the cylinder (association time) and the number of times a lizard moved within $2 \mathrm{~cm}$ of the cylinder (association frequency) during the first trial of the opaque cylinder phase. The opaque cylinder was novel and had never been introduced to the lizards before this point.

To minimize observer bias, blinded methods were used when all behavioral data were recorded and/or analyzed. To test inter-observer reliability, a subset (18\%) of trials (including a random selection of trials from the four tested species) was scored by an independent observer who was not involved in data collection. The independent observer scored trials as described above: correct if the lizard entered the cylinder without touching the surface and incorrect if a touch to the surface occurred before entering the cylinder. Inter-observer reliability was calculated at 0.82 (confidence interval $0.71-0.91$ ) between BS and the independent observer and at 0.86 (confidence interval $0.71-0.89$ ) between $\mathrm{SH}$ and the independent observer. BS and $\mathrm{SH}$ did not score the same videos.

\section{Statistical analyses}

\section{Species-specific performance in the cylinder task}

We investigated if the probability of making a correct detour around the opaque cylinder increased across trials indicative of learning in those individuals that were tested in both test phases. We performed a Bayesian generalized linear mixed model (GLMM; Hadfield 2010) with the detour response (Bernoulli: correct $=1$ or incorrect $=0$ ) given in the opaque cylinder phase as the response variable and trial as the fixed effect. We ran a model for each species separately. Previous work indicates that body condition might affect motivation leading to individual differences in detour performance (Shaw 2017; van Horik et al. 2018). Therefore, we included body condition (scaled mass index after Peig and Green 2009) as an additional fixed effect in each model. Additionally, in sleepy lizards and blue-tongue skinks, we also included sex as a fixed effect because previous work shows that individual differences in performance on detour tasks can be related to sex (Lucon-Xiccato et al. 2019). In sleepy lizards, we also included the origin of animals (wild or captive) as another fixed effect because rearing conditions can affect detour performance (Clarke et al. 1951). We did not include age or origin as a fixed effect in blue-tongue lizards because age was confounded with rearing conditions in this species (for a detailed model description, see Online Resource Table S3).

We investigated if learning occurred across trials in the semi-transparent cylinder phase and if performance shown in the opaque cylinder phase (learning to detour around an opaque cylinder) affected performance in the semitransparent cylinder phase in those individuals that were tested in both test phases. To this end, we extracted average individual learning rates (rate of change in the probability of making a correct detour per trial) shown in the opaque cylinder phase from the random effects posterior for trial. Then, we performed a Bayesian GLMM with the detour response (Bernoulli: correct $=1$ or incorrect $=0$ ) recorded during the semi-transparent cylinder phase as the response variable and trial and individual learning rate (opaque cylinder phase) as fixed effects. Again, we ran a model for each species separately. Additionally, body condition was included as another fixed effect. As before, we included sex as a fixed effect in bluetongue skinks and sex and origin in sleepy lizards. Lastly, preexperience with cognitive assays might affect performance in detour tasks (Kabadayi et al. 2017a). To test for an effect in our lizards, we calculated the sum of trials each individual had performed in a cognitive task before being tested in our experiment and this variable was added as another fixed effect in each model (for a detailed model description, see Online Resource Table S3).

We were interested in whether neophobia had any effect on individual performance (Kabadayi et al. 2017a) in those 
individuals that were tested in both test phases. To measure neophobia, we looked at a lizard's behavior towards the novel cylinder in the first trial of the opaque cylinder phase. We measured trial latency (time in seconds to approach the cylinder) and association time (time in seconds spent within $2 \mathrm{~cm}$ of the cylinder). As we were unsure if these two measures represented the same or different aspects of neophobia, we tested if trial latency and association time were correlated using a Spearman rank correlation. The two variables were not correlated in any of the tested species (Spearman rank correlation, $p=0.289-0.831$; Online Resource Table S4). We, therefore, ran one linear model (LM, Bates et al. 2015) each for trial latency and association time as the response variable separately for each species. In the models with association time as the response variable, we divided association time by the association frequency (the number of times an individual approached the opaque cylinder to within $2 \mathrm{~cm}$ ) to account for baseline activity level. We included individual learning rate as a measure of performance for the opaque cylinder phase and the number of correct detours out of 10 as a measure of performance for the semi-transparent cylinder phase as fixed effects. The models looking at the time to approach the cylinder included trial latency as the response variable and individual learning rate from the opaque cylinder phase and the number of correct detours around the semitransparent cylinder as fixed effects (for a detailed model description, see Online Resource Table S3). To ensure that model residuals conformed to model assumptions of residual normality, we visually inspected qqplots. In some models (see Online Resource Table S3), we log transformed response variables to conform to residual normality.

\section{Between-species comparison in the cylinder task}

Besides the data collected in the current study, data from another species, the eastern water skink (Eulamprus quoyii), were available from a previous study (Szabo et al. 2019b) and included in the between-species analysis. We closely followed procedure and setup in Szabo et al. (2019b) testing lizards in the same environment, same time of day during the lizards' active season, and using the same experimenter (BS) leading all testing. Only data from those individuals (of all tested species) that participated in both phases were included in the following analyses.

To compare performance in the opaque cylinder phase between species, we applied a Bayesian GLMM using the detour response (Bernoulli: correct $=1$ or incorrect $=0$ ) as the response variable and species, and trial and their interaction as fixed effects. Because the model results showed significant differences between species, we wanted to know which species differed significantly. To be able to compare pairs of species, we ran the same model five times, rotating the contrasts of species (contrasts of variable species: water skinks, sleepy lizard, blue-tongue skink, gidgee skink, and tree skink).

To investigate if the performance during the semitransparent cylinder phase differed between species, we again used a Bayesian GLMM. We used the detour response (Bernoulli: correct $=1$ or incorrect $=0$ ) as the response variable; species, trial, and their interaction as fixed effects. Again, model results suggested a significant difference between species, which we examined by running the same model five times, rotating the contrasts for species.

Differences in performance across species might be caused by both cognitive and non-cognitive factors. Because we used the same methods across species, we were able to compare three non-cognitive factors that might cause species differences: the time spent within $2 \mathrm{~cm}$ of the opaque cylinder (divided by the frequency), the time taken to approach the opaque cylinder (trial latency), and body condition (scaled mass index, SMI), across species. Before comparing body condition, we divided each individual's scaled mass index with the average mass of its species in order to be comparable across species. We applied one LM with the time within $2 \mathrm{~cm}$ divided by the frequency as the response variable, one LM with trial latency as the response variable, and one LM with the corrected SMI as the response variable. In all three models, species was the only fixed effect. After visual inspection of residual plots, we log transformed time spent within $2 \mathrm{~cm}$ and trial latency to conform to model assumptions of residual normality. One individual blue-tongue lizard took over $2000 \mathrm{~s}$ to approach the novel opaque cylinder (trial latency), an outlier that affected results when looking at individual-level effects of neophobia on detour performance (see "Results" below). However, this outlier did not affect results in the species comparison and was therefore included in the analyses.

\section{Task-specific inhibitory control}

We were able to correlate learning rate shown in a visual twochoice discrimination reversal with performance shown in the semi-transparent phase of the cylinder task in 11 gidgee skinks (BS, DWAN, MW unpublished data), 6 sleepy lizards (Szabo and Whiting 2020), and 6 eastern blue-tongue skinks (Szabo et al. 2019a) which had participated in both experiments. First, we ran a Bayesian GLMM with first choice of stimulus in the two-choice discrimination task (Bernoulli: correct/rewarded = 1 or incorrect/unrewarded $=0$ ) as the response variable, trial as the only fixed effect, and trial nested in animal identity as the random effect. From the random effect posterior of trial, we extracted the average learning rate (the probability of making a correct choice per trial) for each individual. Next, we used this learning rate as the only fixed effect in a Bayesian GLMM with the detour response (Bernoulli: correct $=1$ or incorrect $=0$ ) given in the semi-transparent cylinder phase as 
the response variable. This was done for each species separately because we were only interested in finding out if individual performance correlated across tasks. We were unable to add the learning rate shown in the reversal task to the main model looking at detour performance in the semi-transparent phase because not all animals were tested in both tasks leading to missing data in this fixed effect.

All statistical analyses were performed in $\mathrm{R}$ version 3.5.3 (R Development Core Team 2008).

We chose to use Bayesian modeling because it is very robust when analyzing non-Gaussian data (Hadfield 2010). All Bayesian models included a random effect of trial nested in animal identity. We did this to account for repeated measures and non-independence of samples and to avoid autocorrelation. For all Bayesian models, we confirmed that no autocorrelation (correlation between lags $<0.1$; Hadfield 2010) and sufficient mixing (by visually inspecting plots of MCMC chains; Hadfield 2010) occurred and that the Markov chain was run long enough and did not show any sampling errors (Heidelberg and Welch diagnostic tests; Hadfield 2010).

\section{Data availability}

All datasets generated and the code used for analyses in the current study are published online (Open Science framework, https://doi.org/10.17605/OSF.IO/CJ6B4).

\section{Results}

\section{Species-specific performance in the cylinder task}

Overall, $60 \%$ (7/12) of tree skinks, 95\% (18/19) of gidgee skinks, $85 \%$ (11/13) of sleepy lizards, and 100\% (12/12) of blue-tongue skinks reached the learning criterion in the opaque cylinder phase and were then tested in the semitransparent cylinder phase (Table 1, Fig. $1 \mathrm{~b}$; the cumulative sum of correct detours around the semi-transparent cylinder for each individual tested are shown in Online Resource Fig. S3).

Only gidgee skinks and sleepy lizards significantly improved their probability of making a correct detour around the opaque cylinder (GLMM, gidgee skinks: trial $=0.185$, $95 \% \mathrm{CI}_{\text {low }}=0.062,95 \% \mathrm{CI}_{\text {up }}=0.313, p=0.0002$; sleepy lizards: trial $=0.933,95 \% \mathrm{CI}_{\mathrm{low}}=0.252,95 \% \mathrm{CI}_{\mathrm{up}}=1.760, p=$ 0.001; Online Resource Table S5) indicative of learning. The sexes did not differ in their performance in the opaque cylinder phase (GLMM, sleepy lizards: male $=0.055,95 \% \mathrm{CI}_{\text {low }}=$ $-2.011,95 \% \mathrm{CI}_{\mathrm{up}}=2.413, p=0.995$; blue-tongue skinks: male $=-0.328,95 \% \mathrm{CI}_{\text {low }}=-1.931,95 \% \mathrm{CI}_{\mathrm{up}}=1.308, p=$ 0.676; Online Resource Table S5) and neither did wild caught and captive sleepy lizards (GLMM, wild $=0.588,95 \%$ $\mathrm{CI}_{\text {low }}=-2.108,95 \% \mathrm{CI}_{\text {up }}=2.850, p=0.549$; Online
Resource Table S5). Performance in the opaque cylinder phase significantly correlated with body condition in bluetongue skinks only (GLMM, body condition $=0.078,95 \%$ $\mathrm{CI}_{\text {low }}=0.012,95 \% \mathrm{CI}_{\text {up }}=0.140, p=0.007$; Online Resource Table S5), showing that individuals in better condition performed better.

In the semi-transparent cylinder phase, neither sex, body condition, pre-experience, nor individual learning rate shown in the opaque cylinder phase significantly predicted detour performance (GLMM, $p>0.05$; Online Resource Table S6). Lizards did not learn to detour around the semi-transparent cylinder (GLMM, $p>0.05$; Online Resource Table S6). However, the estimated learning rate was significantly negative in blue-tongue skinks (GLMM, trial $=-0.255,95 \%$ $\mathrm{CI}_{\text {low }}=-0.442,95 \% \mathrm{CI}_{\text {up }}=-0.072, p=0.007$; Online Resource Table S6) indicating that most animals made correct detours at the start of the test but the probability of making a correct detour decreased over the 10 trials.

Overall, neophobia did not affect task performance within species except in blue-tongue skinks in which trial latency was significantly negatively correlated with learning rate in the opaque cylinder phase (LM, learning rate $=-5526.29, \mathrm{SE}=$ 1005.41, $t=-5.497, p=0.0004)$. This result was, however, caused by an outlier and disappeared after this outlier was removed $(\mathrm{LM}$, learning rate $=-1591.020, \mathrm{SE}=1814.112$, $t=-0.877, p=0.406$; Online Resource Table S7). Otherwise, neither trial latency (the time to approach the novel opaque cylinder) nor association time (time spent within $2 \mathrm{~cm}$ of the novel opaque cylinder divided by the association frequency) were correlated with individual learning rate from the opaque cylinder phase or the number of correct detours performed in the semi-transparent cylinder phase (LM, $p>0.05$; Online Resource Table S7).

\section{Between-species comparison in the cylinder task}

Species differed in their performance during the opaque as well as the semi-transparent cylinder phase. During the opaque cylinder phase, blue-tongue skinks performed significantly better than eastern water skinks (GLMM, blue-tongue skink $=1.478,95 \% \mathrm{CI}_{\text {low }}=0.160,95 \% \mathrm{CI}_{\text {up }}=2.814, p=$ 0.030; Online Resource Table S8). Average learning rates did not differ between species (Online Resource Table S9). Furthermore, blue-tongue skinks performed significantly better than every other lizard species tested in the semitransparent cylinder phase but showed lower learning rates (Table 2).

Time spent within $2 \mathrm{~cm}$ of the opaque cylinder, time to approach the opaque cylinder, and body condition differed across species (Fig. 2a-c; Online Resource Table S10, S11). Tree skinks associated the least amount of time with the novel, opaque cylinder (Fig. 2a; Online Resource Table S10), while sleepy lizards were the fastest to approach the opaque cylinder 
Table 2 Estimates, 95\% confidence intervals (CIs), and significance level $(p)$ of the fixed effect species (left) and its interaction with trial (right) calculated by the models investigating species difference in performance during the semi-transparent cylinder phase. To be able to compare species performance, the model was run five times rotating the fixed effect level. The left species column represents the fixed effect level to which each other level (top row species) was compared. Values within bold boxes represent the intercept of each model. Significant comparisons are highlighted in gray. Light gray $<0.05$, medium gray $<0.01$, dark gray $<0.001$. Significant intercepts are not highlighted

\begin{tabular}{|c|c|c|c|c|c|c|c|c|c|c|c|}
\hline \multirow{2}{*}{\multicolumn{2}{|c|}{\begin{tabular}{|r|} 
Estimate \\
Cls \\
$p$
\end{tabular}}} & \multicolumn{5}{|c|}{ Main fixed effect levels (species) } & \multicolumn{5}{|c|}{ Species effect levels interaction with trial } \\
\hline & & Water skink & $\begin{array}{l}\text { Sleepy } \\
\text { lizard }\end{array}$ & $\begin{array}{l}\text { Blue-tongue } \\
\text { skink }\end{array}$ & $\begin{array}{l}\text { Gidgee } \\
\text { skink }\end{array}$ & Tree skink & Water skink & $\begin{array}{l}\text { Sleepy } \\
\text { lizard }\end{array}$ & $\begin{array}{c}\text { Blue-tongue } \\
\text { skink }\end{array}$ & $\begin{array}{l}\text { Gidgee } \\
\text { skink }\end{array}$ & Tree skink \\
\hline \multirow{5}{*}{ 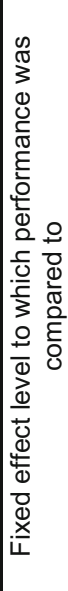 } & Water skink & $\begin{array}{c}-0.650 \\
-1.515- \\
0.202 \\
0.132\end{array}$ & $\begin{array}{c}-0.520 \\
-1.906- \\
0.851 \\
0.455\end{array}$ & $\begin{array}{c}1.671 \\
0.408- \\
3.032 \\
0.013\end{array}$ & $\begin{array}{c}-1.223 \\
-2.494- \\
0.027 \\
0.060\end{array}$ & $\begin{array}{c}-0.963 \\
-2.671- \\
0.653 \\
0.246\end{array}$ & $\begin{array}{c}-0.650 \\
-1.515- \\
0.202 \\
0.132\end{array}$ & $\begin{array}{c}0.025 \\
-0.203- \\
0.252 \\
0.832\end{array}$ & $\begin{array}{c}-0.262 \\
-0.486- \\
-0.043 \\
0.023\end{array}$ & $\begin{array}{c}0.076 \\
-0.137- \\
0.266 \\
0.460\end{array}$ & $\begin{array}{c}0.070 \\
-0.205- \\
0.335 \\
0.606\end{array}$ \\
\hline & Sleepy lizard & $\begin{array}{c}0.535 \\
-0.767- \\
2.054 \\
0.459\end{array}$ & $\begin{array}{l}-1.186 \\
-2.262- \\
-0.077 \\
0.033\end{array}$ & $\begin{array}{c}2.224 \\
0.695- \\
3.661 \\
0.002\end{array}$ & $\begin{array}{c}-0.702 \\
-2.033- \\
0.821 \\
0.333\end{array}$ & $\begin{array}{c}-0.391 \\
-2.155- \\
1.369 \\
0.665\end{array}$ & $\begin{array}{c}-0.028 \\
-0.261- \\
0.198 \\
0.817\end{array}$ & $\begin{array}{c}-1.186 \\
-2.262- \\
-0.077 \\
0.033\end{array}$ & $\begin{array}{l}-0.293 \\
-0.540- \\
-0.047 \\
0.018\end{array}$ & $\begin{array}{c}0.048 \\
-0.183- \\
0.271 \\
0.674\end{array}$ & $\begin{array}{c}0.038 \\
-0.244- \\
0.332 \\
0.795\end{array}$ \\
\hline & $\begin{array}{l}\text { Blue-tongue } \\
\text { skink }\end{array}$ & $\begin{array}{l}-1.683 \\
-2.955- \\
-0.323 \\
0.011\end{array}$ & $\begin{array}{c}-2.235 \\
-3.674- \\
-0.647 \\
0.004\end{array}$ & $\begin{array}{c}1.035 \\
-0.014- \\
1.977 \\
0.038\end{array}$ & $\begin{array}{c}-2.913 \\
-4.326- \\
-1.582 \\
0.0002 \\
\end{array}$ & $\begin{array}{c}-2.624 \\
-4.440- \\
-0.972 \\
0.001\end{array}$ & $\begin{array}{c}0.264 \\
0.045- \\
0.490 \\
0.017\end{array}$ & $\begin{array}{c}0.293 \\
0.052- \\
0.547 \\
0.019\end{array}$ & $\begin{array}{c}1.035 \\
-0.014- \\
1.977 \\
0.038\end{array}$ & $\begin{array}{c}0.340 \\
0.110- \\
0.554 \\
0.002\end{array}$ & $\begin{array}{c}0.330 \\
0.035- \\
0.602 \\
0.021\end{array}$ \\
\hline & Gidgee skink & $\begin{array}{c}1.233 \\
-0.034- \\
2.482 \\
0.055\end{array}$ & $\begin{array}{c}0.695 \\
-0.700- \\
2.159 \\
0.332\end{array}$ & $\begin{array}{c}2.926 \\
1.556- \\
4.342 \\
0.0002\end{array}$ & $\begin{array}{c}-1.887 \\
-2.832- \\
-0.964 \\
0.0002\end{array}$ & $\begin{array}{c}0.297 \\
-1.411- \\
1.960 \\
0.731\end{array}$ & $\begin{array}{c}-0.075 \\
-0.277- \\
0.133 \\
0.467\end{array}$ & $\begin{array}{c}-0.047 \\
-0.283- \\
0.175 \\
0.682\end{array}$ & $\begin{array}{c}-0.341 \\
-0.571- \\
-0.117 \\
0.003\end{array}$ & $\begin{array}{c}-1.887 \\
-2.832- \\
-0964 \\
0.0002\end{array}$ & $\begin{array}{c}-0.009 \\
-0.268- \\
0.258 \\
0.942\end{array}$ \\
\hline & Tree skink & $\begin{array}{c}0.949 \\
-0.726- \\
2.610 \\
0.264\end{array}$ & $\begin{array}{c}0.408 \\
-1.368- \\
2.180 \\
0.654\end{array}$ & $\begin{array}{c}2.640 \\
0.842- \\
4.290 \\
0.002\end{array}$ & $\begin{array}{c}-0.287 \\
-1.996- \\
1.369 \\
0.741\end{array}$ & $\begin{array}{c}-1.598 \\
-2.940- \\
-0.112 \\
0.022\end{array}$ & $\begin{array}{c}-0.066 \\
-0.334- \\
0.203 \\
0.641\end{array}$ & $\begin{array}{c}-0.037 \\
-0.325- \\
0.249 \\
0.812\end{array}$ & $\begin{array}{c}-0.331 \\
-0.614- \\
-0.045 \\
0.022\end{array}$ & $\begin{array}{c}0.01 \\
-0.261- \\
0.277 \\
0.937\end{array}$ & $\begin{array}{c}-1.598 \\
-2.940- \\
-0.112 \\
0.022\end{array}$ \\
\hline
\end{tabular}

(Fig. 2a; Online Resource Table S10). Blue-tongue skinks had the lowest body condition (Fig. 2c) and significantly differed from all other species in body condition (Online Resource Table S11).

\section{Task-specific inhibitory control}

Individual-level performance detouring around a semitransparent cylinder did not correlate with the learning rate shown in a visual two-choice discrimination task (GLMM, gidgee skinks: reversal learning rate $=11.318, \mathrm{CI}_{\text {low }}=-$ $29.830,95 \% \mathrm{CI}_{\text {up }}=54.908, p=0.597$; sleepy lizards: reversal learning rate $=1.481, \mathrm{CI}_{\mathrm{low}}=-102.095,95 \% \mathrm{CI}_{\mathrm{up}}=111.181$, $p=0.997$; blue-tongue skinks: reversal learning rate $=8.011$, $\mathrm{CI}_{\text {low }}=-46.346,95 \% \mathrm{CI}_{\text {up }}=61.893, p=0.748$; Online Resource Table S12).

\section{Discussion}

Individuals from all tested species reached our predetermined learning criterion in the opaque cylinder phase and completed 10 trials of detouring to the side of a semi-transparent mesh cylinder to retrieve a visible reward from within. Gidgee skinks and sleepy lizards learnt to detour to the side openings of an opaque cylinder. In the opaque cylinder phase, neither sex, animal origin, nor neophobia predicted individual differences in task performance in any of the tested species, but blue-tongue lizards in better body condition performed better.
In the semi-transparent cylinder phase, none of the tested variables (sex, origin, neophobia, body condition, learning rate in the opaque cylinder phase, or pre-experience with other cognitive tasks) predicted individual differences in performance in any of the tested species. Interestingly, blue-tongue skinks correctly retrieved the reward from within the mesh cylinder (without touching its surface) more often at the beginning of the semi-transparent phase but did make more errors in later trials. Furthermore, blue-tongue skinks also performed better than water skinks in the opaque cylinder phase and outperformed all other tested species during the semitransparent cylinder phase. Neophobia as well as body condition differed across species. Tree skinks spent the least time close to the cylinder and sleepy lizards approached the cylinder the fastest in the first trial of the opaque cylinder phase. Blue-tongue skinks had the lowest body condition, possibly leading to greater motivation to reach the food reward within the cylinder which could drive the difference in cylinder task performance we found across species. Finally, individuallevel measures of inhibition were not associated across tasks, suggesting that different inhibitory skills are involved in detour success and discrimination reversal learning.

\section{Species-specific performance in the cylinder task}

As expected, lizards were able to detour around an opaque cylinder as well as a semi-transparent cylinder. Only gidgee skinks and sleepy lizards learned to do so, and only in the opaque cylinder phase. Neither sex, animal origin, nor 
neophobia affected performance in the opaque cylinder phase. However, blue-tongue lizards' performance was influenced by body condition: individuals in better condition made less contact with the surface of the opaque cylinder (less errors). A similar result was reported for North Island robins tested in a transparent cylinder task (Shaw 2017). Contrary to previous studies, individual performance in the semi-transparent cylinder phase was neither affected by animal origin (Clarke et al. 1951) or body condition (Shaw 2017). We found no effect of sex on performance detouring the semi-transparent cylinder, which is similar to what has been reported for pheasant chicks (van Horik et al. 2018) but contrasts with results found in guppies (Lucon-Xiccato et al. 2019). Furthermore, similar to previous studies, we found no effect of neophobia (Stow et al. 2018; van Horik et al. 2018) or pre-experience (Shaw 2017; Santacà et al. 2019b) on individual task performance. Importantly, none of the tested species learnt to detour a semi-transparent cylinder. Detour training using an opaque cylinder is designed to reduce the influence learning and problem solving have on detour performance in the subsequent transparent cylinder test phase to reliably measure inhibitory ability (Kabadayi et al. 2017a, b). It is still to be determined if our lizards had shown learning without the previous training with the opaque cylinder. Furthermore, dogs without previous training on an opaque cylinder performed poorly when detouring to the side of a transparent cylinder (Vernouillet et al. 2018). Whether lizards show a similar reduced performance without prior detour training remains to be determined. Overall, the differences and similarities across study findings suggest different factors influence cylinder task performance across species. Many studies suffer from small sample sizes caused by limited availability of individuals for testing; ours is no exception. It has been suggested that to reliably detect individual differences a minimum of 40 individuals producing a total of 1000 observations is required (van de Pol 2012). It is possible that subtle individual differences in cylinder task performance might exist in our lizard species, but our sample sizes (and resulting effect sizes) might be too small to detect them.

The learning performance during the opaque cylinder phase did not predict performance in the semi-transparent cylinder phase in any of the tested lizard species and this is similar to what has been found in dogs (Canis familiaris; Bray et al. 2014) and douc langurs (Pygathrix nemaeus and $P$. cinereal, Rudolph and Fichtel 2017). Although we found no correlation between test phases, blue-tongue skinks were the only species (out of 5) to make correct choices right at the start of the semi-transparent cylinder phase. All individual blue-tongue skinks made a correct choice within their first two trials. To make such correct choices, it is possible that these lizards had transferred knowledge about how to detour to the side openings from the opaque cylinder. At the start of the semi-transparent cylinder phase, blue-tongue skinks had no prior knowledge of how to solve this task except for what they had learnt in the previous stage. Further tests are, however, necessary to confirm that blue-tongue skinks can transfer knowledge between phases.

\section{Differences between species in the cylinder task}

The performance during the semi-transparent cylinder phase differed across species. Blue-tongue skinks consistently outperformed the other four species while none of the other four species significantly differed from each other in performance. Our analyses of neophobia and body condition show species-level differences. We measured neophobia as the behavior shown by our lizards towards a novel object (the
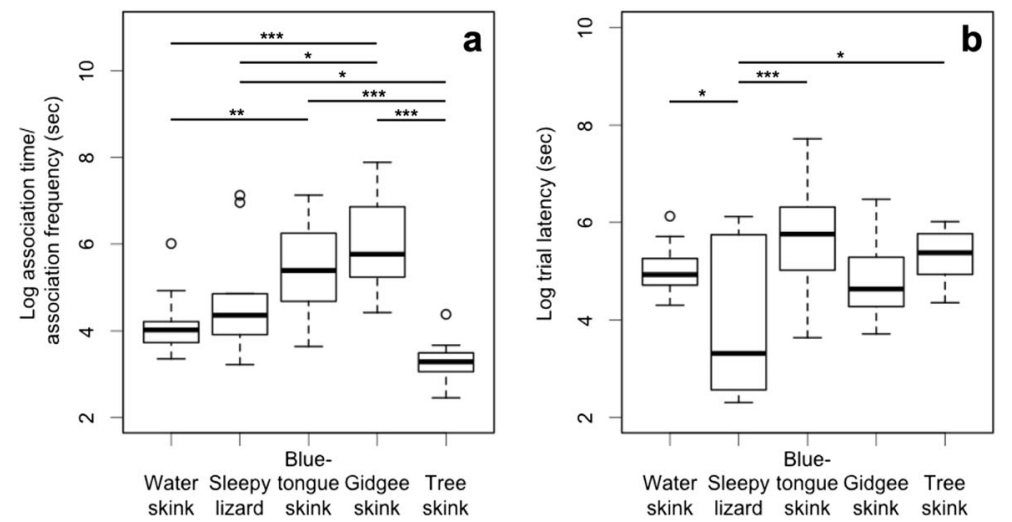

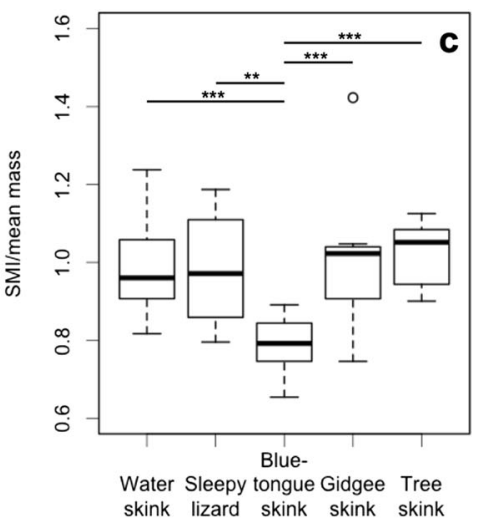

sec; log transformed) from trial start to when the cylinder was first touched across the tested species. $\mathbf{c}$ Box plot showing median (bold line), quartile ( $50 \%$ of data within the box), and the maximum and minimum valies (edge of whiskers) excluding outliers (circles) of body condition (scaled mass index (SMI) divided by the average mass of species) across the tested species $*<0.05$, $* *<0.01, * * *<0.001$ (created with $\mathrm{R}$ base plot and modified in Microsoft PowerPoint) 
opaque cylinder) in a familiar environment. The time to approach the novel object and the time spent within $2 \mathrm{~cm}$ of the object were uncorrelated suggesting that they measure different aspects of neophobia. Only the results from comparing body condition across species, however, show a pattern consistent with the differences in cylinder task performance. Blue-tongue skinks had the lowest body condition compared to all other species, and none of the other species differed from each other. This difference in body condition might have caused motivational changes in blue-tongue lizards and resulted in them performing better as a group than the other four lizard species. It is therefore possible that, if body condition would have been equal across species, no species differences in task performance would have occurred. At this point, we are unable to determine if motivation alone led to our result or if blue-tongue skinks possess better inhibitory control ability as measured by our task. Furthermore, because our analyses show no significant increase in the probability of making a correct detour across the 10 test trials, we can exclude differences in learning ability as an explanation for differences in task performance among species. Our result highlights, however, that non-cognitive factors should always be considered when task performance is compared across individuals as well as species.

When looking at average score (mean correct detours across the 10 test trials), blue-tongue skinks received the highest score, followed by water skinks, sleepy lizards, tree skinks, and gidgee skinks. In primates, superior inhibitory performance positively correlated with the number of food categories within the diet (e.g., MacLean et al. 2014) such that folivorous species scored the lowest (MacLean et al. 2014; Rudolph and Fichtel 2017). This result is partially consistent with the "Ecological Intelligence Hypothesis" (Gibson 1986; Byrne 1997; Rosati 2017), in which factors such as feeding ecology underlie the evolution of enhanced cognitive abilities involved in, for example, extractive foraging or hunting (Gibson 1986; Byrne 1997; Rosati 2017). In hyenas, individuals from larger social groups performed better on the cylinder task (Johnson-Ulrich and Holekamp 2020) consistent with the "Social Intelligence hypothesis," under which the challenges of social group life drives the evolution of larger brains and associated cognitive abilities to keep track of conspecifics and relationships and exercise appropriate social cognitive skill to manage these relationships (Jolly 1966; Humphrey 1976; Byrne and Whiten 1988; Dunbar 1998). Our sample of lizards showed marked differences in diet composition and sociality. Although omnivorous, gidgee skinks consume mostly (> 80\%; Duffield and Bull 1998) plant material while water skinks are insectivorous (Veron 1969) (Online Resource Table S1). Regarding sociality, gidgee skinks live in monogamous, kin-based family groups including multiple adults (Chapple 2003), tree skinks also live in family groups, albeit nuclear families which include offspring of the same or previous season (Chapple 2003; O'Connor and Shine 2003), sleepy lizards show long-term monogamy but no association between adults and their offspring (Bull et al. 2017), and water skinks and blue-tongue lizards are solitary (Koenig et al. 2001; Cogger 2014) (Online Resource Table S1). We conducted two preliminary analyses to test if feeding ecology and/or sociality could be possible causes for the species differences we found (Online Resource). Our preliminary analyses (Online Resource) indicate that more herbivorous lizard species received lower inhibition scores (correct detours out of 10) compared to species with more protein-rich diets consistent with the "Ecological Intelligence Hypothesis." Furthermore, we found a strong negative correlation between score and group size indicating that more social species received lower scores inconsistent with the "Social Intelligence hypothesis." Because of our low sample size (five species), feeding ecology and sociality are confounded; the most herbivorous species was also the most social. Data on a wider range of lizard species as well as additional data from the already tested species is required to test if feeding ecology and/or sociality (among other traits not considered here) can lead to differences in performance across lizard species.

Besides differences in performance, we experienced differences in drop-out rate in the opaque cylinder phase; not all individuals tested reached the learning criterion within 30 trials (Online Resource Table S2), with the exception of the blue-tongue skinks. The highest drop-out rate was in tree skinks (42\%), followed by water skinks (20\%), sleepy lizards $(15 \%)$, and finally, gidgee skinks $(5 \%)$. These drop-out rates could have been caused by non-cognitive factors including differential reliance on food chemicals during foraging or food motivation. For example, zebrafish (Danio rerio) outperformed three other fish species in a cylinder task although additional tests showed that zebrafish used odor cues to their advantage to solve the task. When such odor cues were eliminated, the species difference in performance disappeared (Santacà et al. 2019a). Guppies, however, do not rely on olfactory cues to solve cylinder detour tasks (Santacà et al. 2019b). The pattern shown by our lizards is inconsistent with the level of reliance on food chemicals. Sleepy lizards, which showed a $15 \%$ drop-out rate, rely on food chemicals when foraging (Cooper 2000) and should perform better while gidgee skinks (5\% drop) are sit-and-wait foragers (relying more heavily on vision during foraging) and should have shown a greater drop-out rate than sleepy lizards. Therefore, it seems unlikely that vomerolfaction alone affected the success rate. Furthermore, with more than 30 trials, all individuals might have eventually reached the learning criterion. Although cognitive differences could partly explain the differences in drop-out rate between species, non-cognitive factors cannot be ruled out at this stage. 


\section{Task-specific inhibitory control}

Our results add to the growing body of evidence that individual performance on different cognitive tasks testing for inhibitory control do not correlate with each other. Detour tasks around differently shaped barriers likely require motor response inhibition (inhibition of prepotent responses towards an external lure = the reward; Dias et al. 1996), while reversal learning probably mainly involves stimulus response inhibition, a different contextual sub-class of inhibitory control (Dias et al. 1996). Exercising control over a stimulus-reward association requires ignoring one stimulus in favor of another stimulus for which no such association has yet formed. In common marmosets (Callithrix jacchus), lesions to the orbital prefrontal cortex selectively impaired the first reversal of a visual two-choice discrimination (impaired stimulus-reward inhibition), while lesions to the lateral prefrontal cortex selectively impaired the first shift to a second stimulus dimension (attentional inhibition; Dias et al. 1997). A follow-up experiment indicated that the impairment was due to issues with inhibitory control while associative learning was not affected (Dias et al. 1997). In painted turtles (Chrysemys picta), lesions to the dorsal cortex impaired reversal learning and attentional set-shifting but not the acquisition of a discrimination in a visual two-choice discrimination task (Cranney and Powers 1983). In a visual go-no-go task, however, lesions to the dorsal cortex did affect acquisition and reversal performance in these turtles (Grisham and Powers 1989). The results in monkeys clearly demonstrate that different neural substrates underlie inhibitory control of stimulus-reward relationships (reversal task) and attentional inhibition (attentional set-shifting) of visual information. In turtles, the same neural substrate, the dorsal cortex, seems important for inhibitory control in general. The study in monkeys, however, targeted specific areas within the prefrontal cortex while the turtle studies targeted a larger brain area. It is therefore possible that specific areas within the dorsal cortex are associated with processing different sub-classes of inhibitory control. We are unaware of any study investigating the involvement of different neural substrates in visual discrimination and reversal learning or motor response inhibition in lizards. The lack of individual-level correlation between tasks suggests different processing units for the different sub-classes of inhibitory control in lizards. Aside from different inhibitory skills, tasks might require additional cognitive abilities for successful completion. A good memory of the correct motor response, for example, might greatly enhance performance in a detour task while a strong memory of the previous stimulus-reward relationship might greatly affect reversal learning. To better understand how inhibitory control is exercised in different cognitive tasks, research should focus on investigating how these tasks are accomplished at a neural level.
In summary, we show that our modified version of the cylinder task is a suitable test of motor response inhibition to be used in different lizard species. Although our conclusions are limited due to low sample sizes across and within species, our results demonstrate the effects of non-cognitive factors in task performance and suggest the need for further investigations into the cognitive and non-cognitive factors involved in creating these differences. Moreover, our results strengthen the conclusion that inhibition scores earned in different cognitive tasks do not correlate, demonstrating that this area of research deserves further study. Taken together, our study provides insight into inhibitory control across multiple lizard species and adds valuable data to the growing knowledge base on animal inhibitory control abilities. Significantly, due to the phylogenetic relationship of reptiles to birds and mammals (reptiles split from mammals $\sim 320$ million years ago and lizards split from birds 280 million years ago; Alföldi et al. 2011), data such as that presented here can help us better understand the evolution of cognitive skills and help to identify predictors of individual and species differences in cognitive performance across taxa.

\section{Ethical note}

The experiments performed in this study were strictly noninvasive observations of behavior. In fact, our trials were a form of behavioral enrichment because animals had the choice to engage with a task in order to get a food reward. Experimental procedures followed the guidelines outlined by the Association for the Study of Animal Behaviour/Animal Behavior Society for the use of animals in research (Guidelines for the treatment of animals in behavioral research and teaching 2018) and were approved by the Macquarie University Animal Ethics Committee (ARA \# 2013/031). Wild lizards were hand-captured and kept individually in a temperature-controlled environment until transported to Macquarie University by car in cloth bags. Animal collection from the wild was approved by the New South Wales National Parks and Wildlife Service (OEH; License \#SL101972). At the end of experiments, animals were rehomed following OEH guidelines.

Acknowledgments We thank Anthony Stimson and Julia Riley for providing lizards, Bruno Pleno, Faustine Degottex, Isabel Damas, Rob Ambrose, and Victor Frichot for their help catching skinks, Levin Wiedenroth for his help collecting data, and Jonathan Ogle for blind coding.

Author contributions Conceptualization: BS, MW. Data curation: BS. Formal Analysis: BS. Funding acquisition: BS, MW. Investigation: BS, SH. Methodology: BS, MW. Project administration: BS, MW. Resources: MW. Supervision: MW. Validation: BS, MW. Visualization: BS. Writing — original draft: BS, SH. Writing—review and editing: BS, SH, MW. 
Funding The study was funded by the Australian Society of Herpetologists (Student research grant to BS) and Macquarie University (iMQRes scholarship and Rice Memorial Field Research Award received by BS). Open Access charges were covered by the University of Bern.

\section{Compliance with ethical standards}

Conflict of interest The authors declare that they have no conflict of interest.

Ethical approval All applicable international, national, and/or institutional guidelines for the care and use of animals were followed. All procedures performed in studies involving animals were in accordance with the ethical standards of the institution or practice at which the study was conducted.

Open Access This article is licensed under a Creative Commons Attribution 4.0 International License, which permits use, sharing, adaptation, distribution and reproduction in any medium or format, as long as you give appropriate credit to the original author(s) and the source, provide a link to the Creative Commons licence, and indicate if changes were made. The images or other third party material in this article are included in the article's Creative Commons licence, unless indicated otherwise in a credit line to the material. If material is not included in the article's Creative Commons licence and your intended use is not permitted by statutory regulation or exceeds the permitted use, you will need to obtain permission directly from the copyright holder. To view a copy of this licence, visit http://creativecommons.org/licenses/by/4.0/.

\section{References}

Alföldi J, Di Palma F, Grabherr M et al (2011) The genome of the green anole lizard and a comparative analysis with birds and mammals. Nature 477:587-591

Anderson RC, Searcy WA, Peters S, Hughes M, DuBois AL, Nowicki S (2016) Song learning and cognitive ability are not consistently related in a songbird. Anim Cogn 20:309-320. https://doi.org/10. 1007/s10071-016-1053-7

Bates D, Maechler M, Bolker B, Walker S (2015) Fitting linear mixedeffects models using lme4. J Stat Softw 67:1-48. https://doi.org/10. 18637/jss.v067.i01

Bobrowicz K, Osvath M (2018) Cats parallel great apes and corvids in motor self-regulation - not brain but material size matters. Front Psychol 9:1995. https://doi.org/10.3389/fpsyg.2018.01995

Bojczyk KE, Corbetta D (2004) Object retrieval in the 1st year of life: learning effects of task exposure and box transparency. Dev Psychol 40:54-66. https://doi.org/10.1037/0012-1649.40.1.54

Boogert NJ, Anderson RC, Peters S, Searcy WA, Nowicki S (2011) Song repertoire size in male song sparrows correlates with detour reaching, but not with other cognitive measures. Anim Behav 81: 1209-1216

Bray EE, MacLean EL, Hare BA (2014) Context specificity of inhibitory control in dogs. Anim Cogn 17:15-31. https://doi.org/10.1007/ s10071-013-0633-z

Brucks D, Marshall-Pescini S, Range F (2018) Dogs and wolves do not differ in their inhibitory control abilities in a non-social test battery. Anim Cogn 22:1-15. https://doi.org/10.1007/s10071018-1216-9

Bull CM (1995) Population ecology of the sleepy lizard, Tiliqua rugosa, at Mt Mary, South Australia. Aust J Ecol 20:393-402
Bull CM, Pamula Y (1996) Sexually dimorphic head sizes and reproductive success in the sleepy lizard Tiliqua rugosa. J Zool 240:511-521

Bull CM, Gardner MG, Sih A, Spiegel O, Godfrey SS, Leu ST (2017) Why Is social behavior rare in reptiles? Lessons from sleepy lizards. Adv Study Behav 49:1-26

Burghardt GM (1978) Learning processes in reptiles: detour problems and delayed reactions. In: Gans C, Tinkle DW (eds) Biology of the Reptilia, vol. 7. Ecology and Behaviour A. Academic Press, London, pp 610-614

Byrne RW (1997) The technical intelligence hypothesis: an additional evolutionary stimulus to intelligence? In: Whiten A, Byrne RW (eds) Machiavellian Intelligence II: Extensions and Evaluations. Cambridge University Press, Cambridge, pp 289-311

Byrne RW, Whiten A (1988) Machiavellian intelligence: social expertise and the evolution of intellect in monkeys, apes, and humans. Oxford University Press, Oxford

Chapple DG (2003) Ecology, life-history, and behavior in the Australian Scincid genus Egernia, with comments on the evolution of complex sociality in lizards. Herpetol Monogr 17:145-180

Clarke RS, Heron W, Fetherstonhaugh ML, Forgays DG, Hebb DO (1951) Individual differences in dogs: preliminary report on the effects of early experience. Can J Psychol 5:150-156

Cogger HG (2014) Reptiles and amphibians of Australia, 7th edn. CSIRO Publishing, Victoria

Cooper WE Jr (2000) Food chemical discriminations by the omnivorous Scincid lizards Tiliqua scincoides and Tiliqua rugosa. Herpetologica 56:480-488

Cranney J, Powers AS (1983) The effects of core nucleus and cortical lesions in turtles on reversal and dimensional shifting. Physiol Psychol 11:103-111. https://doi.org/10.3758/BF03326779

Diamond A (1981) Retrieval of an object from an open box: the development of visual-tactile control of reaching in the first year of life. Soc Res Child Dev Abstr 3:78

Diamond A (1990) Developmental time course in human infants and infant monkeys, and the neural bases of inhibitory control in reaching. Ann N Y Acad Sci 608:637-676

Diamond A (2013) Executive functions. Annu Rev Psychol 64:135-168. https://doi.org/10.1146/annurev-psych-113011-143750

Dias R, Robbins TW, Roberts AC (1996) Primate analogue of the Wisconsin card sorting test - effects of excitotoxic lesions of the prefrontal cortex in the marmoset. Behav Neurosci 110:872-886

Dias R, Robbins TW, Roberts AC (1997) Dissociable forms of inhibitory control within prefrontal cortex with an analog of the Wisconsin card sort test - restriction to novel situations and independence from “on-line" processing. J Neurosci 17:9285-9297

Ducatez S, Audet JN, Lefebvre L (2019) Speed-accuracy trade-off, detour reaching and response to PHA in Carib grackles. Anim Cogn 22: 625-633. https://doi.org/10.1007/s10071-019-01258-1

Duffield GA, Bull CM (1998) Seasonal and ontogenetic changes in the diet of the Australian skink Egernia stokesii. Herpetologica 54:414-419

Dunbar RIM (1998) The social brain hypothesis. Evol Anthropol 6:178-190

Fagnani J, Barrera G, Carballo F, Bentosela M (2016) Is previous experience important for inhibitory control? A comparison between shelter and pet dogs in A-not-B and cylinder tasks. Anim Cogn 19: 1165-1172. https://doi.org/10.1007/s10071-016-1024-z

Gardner MG, Hugall AF, Donnellan SC, Hutchinson MN, Foster R (2008) Molecular systematics of social skinks- phylogeny and taxonomy of the Egernia group (Reptilia- Scincidae). Zool J Linnean Soc 154:781-794

Gibson KR (1986) Cognition, brain size, and the extraction of embedded food resources. In: Else JG, Lee PC (eds) Primate ontogeny, cognition, and social behaviour. Cambridge University Press, Cambridge, pp 93-105 
Grisham W, Powers AS (1989) Function of the dorsal and medial cortex of turtles in learning. Behav Neurosci 103:991-997. https://doi.org/ 10.1037/0735-7044.103.5.991

Guidelines for the treatment of animals in behavioural research and teaching (2018) Anim Behav 135:I-X. doi:https://doi.org/10.1016/j. anbehav.2017.10.001

Hadfield JD (2010) MCMC methods for multi-response generalised linear mixed models: The MCMCglmm R package. J Stat Softw 33:122

Humphrey N (1976) The social function of intellect. In: Bateson PPG, Hinde RA (eds) Growing Points in Ethology. Cambridge University Press, Cambridge, pp 303-317

Isaksson E, Utku Urhan A, Brodin A (2018) High level of self-control ability in a small passerine bird. Behav Ecol Sociobiol 72:118. https://doi.org/10.1007/s00265-018-2529-z

Johnson-Ulrich L, Holekamp KE (2020) Group size and social rank predict inhibitory control in spotted hyaenas. Anim Behav 160:157168. https://doi.org/10.1016/j.anbehav.2019.11.020

Jolly A (1966) Lemur social behavior and primate intelligence. Proc Am Assoc Adv Sci 153:501-506

Junghans AF, Sterck EH, Overduin de Vries A, Evers C, De Ridder DT (2016) Defying food - how distance determines monkeys' ability to inhibit reaching for food. Front Psychol 7:158. https://doi.org/10. 3389/fpsyg.2016.00158

Juszczak GR, Miller M (2016) detour behavior of mice trained with transparent, semitransparent and opaque barriers. PLoS One 11:e0162018. https://doi.org/10.1371/journal.pone. 0162018

Kabadayi C, Taylor LA, von Bayern AM, Osvath M (2016) Ravens, New Caledonian crows and jackdaws parallel great apes in motor selfregulation despite smaller brains. R Soc Open Sci 3:160104. https:// doi.org/10.1098/rsos.160104

Kabadayi C, Bobrowicz K, Osvath M (2017a) The detour paradigm in animal cognition. Anim Cogn 21:21-35. https://doi.org/10.1007/ s10071-017-1152-0

Kabadayi C, Krasheninnikova A, O'Neill L, van de Weijer J, Osvath M, von Bayern AMP (2017b) Are parrots poor at motor self-regulation or is the cylinder task poor at measuring it? Anim Cogn 20:11371146. https://doi.org/10.3389/fpsyg.2017.02100

Koenig J, Shine R, Shea G (2001) The ecology of an Australian reptile icon: how do blue-tongued lizards (Tiliqua scincoides) survive in suburbia? Wildl Res 28:215-227

Langkilde T, Shine R (2006) How much stress do researchers inflict on their study animals? A case study using a scincid lizard, Eulamprus heatwolei. J Exp Biol 209:1035-1043. https://doi.org/10.1242/jeb. 02112

Lucon-Xiccato T, Gatto E, Bisazza A (2017) Fish perform like mammals and birds in inhibitory motor control tasks. Sci Rep 7:13144. https:// doi.org/10.1038/s41598-017-13447-4

Lucon-Xiccato T, Gatto E, Bisazza A, Nelson X (2019) Male and female guppies differ in problem-solving abilities. Cur Zool 19:733-744. https://doi.org/10.1093/cz/zoz017

MacLean EL, Hare B, Nunn CL et al (2014) The evolution of self-control. Proc Natl Acad Sci USA 111:E2140-E2148. https://doi.org/10. 1073/pnas.1323533111

Marshall-Pescini S, Virányi Z, Range F (2015) The effect of domestication on inhibitory control: wolves and dogs compared. PLoS One 10:e0118469. https://doi.org/10.1371/journal.pone. 0118469

O'Connor D, Shine R (2003) Lizards in 'nuclear families': a novel reptilian system in Egernia saxatilis (Scincidae). Mol Ecol 12: $743-752$

Peig J, Green AJ (2009) New perspectives for estimating body condition from mass/length data: the scaled mass index as an alternative method. Oikos 118:1883-1891
Phillips CA, Roffey JB, Hall E, Johnson R (2016) Sex identification in the eastern blue-tongued lizard (Tiliqua scincoides White, ex Shaw, 1790) using morphometrics. Aust Vet J 94:256-259. https://doi.org/ 10.1111/avj.12429

R Development Core Team (2008) R: a language and environment for statistical computing. R Foundation for Statistical Computing, Vienna http://www.R-project.org

Regolin L, Vallortigara G, Zanforlin M (1994) Perceptual and motivational aspects of detour behaviour in young chicks. Anim Behav 47: $123-131$

Rosati AG (2017) Foraging cognition: reviving the ecological intelligence hypothesis. Trends Cogn Sci 21:691-702

Rudolph K, Fichtel C (2017) Inhibitory control in douc langurs (Pygathrix nemaeus and P. cinerea). Vietnamese J Primatol 2:7381

Santacà M, Busatta M, Lucon-Xiccato T, Bisazza A (2019a) Sensory differences mediate species variation in detour task performance. Anim Behav 155:153-162. https://doi.org/10.1016/j.anbehav. 2019.05.022

Santacà M, Busatta M, Savașçı BB, Lucon-Xiccato T, Bisazza A (2019b) The effect of experience and olfactory cue in an inhibitory control task in guppies, Poecilia reticulata. Anim Behav 151:1-7. https:// doi.org/10.1016/j.anbehav.2019.03.003

Santos LR, Ericson BN, Hauser MD (1999) Constraints on problem solving and inhibition: object retrieval in cotton-top tamarins (Saguinus oedipus oedipus). J Comp Psychol 113:186-193

Shaw RC (2017) Testing cognition in the wild: factors affecting performance and individual consistency in two measures of avian cognition. Behav Process 134:31-36. https://doi.org/10.1016/j.beproc. 2016.06.004

Stow MK, Vernouillet A, Kelly DM (2018) Neophobia does not account for motoric self-regulation performance as measured during the detour-reaching cylinder task. Anim Cogn 21:565-574. https://doi. org/10.1007/s10071-018-1189-8

Szabo B, Whiting MJ (2020) Do lizards have enhanced inhibition? A test in two species differing in ecology and sociobiology. Behav Process 172:104043. https://doi.org/10.1016/j.beproc. 2020.104043

Szabo B, Noble DWA, Byrne RW, Tait DS, Whiting MJ (2018) Subproblem learning and reversal of a multidimensional visual cue in a lizard: evidence for behavioural flexibility? Anim Behav 144: $17-26$

Szabo B, Noble DWA, Byrne RW, Tait DS, Whiting MJ (2019a) Precocial juvenile lizards show adult-level learning and behavioural flexibility. Anim Behav 154:75-84. https://doi.org/10.1016/j. anbehav.2019.06.003

Szabo B, Noble DWA, Whiting MJ (2019b) Context-specific response inhibition and differential impact of a learning bias in a lizard. Anim Cogn 22:217-329. https://doi.org/10.1007/s10071-019-01245-6

van de Pol M (2012) Quantifying individual variation in reaction norms: how study design affects the accuracy, precision and power of random regression models. Methods Ecol Evol 3:268-280. https://doi. org/10.1111/j.2041-210X.2011.00160.x

van Horik JO, Madden JR (2016) A problem with problem solving: motivational traits, but not cognition, predict success on novel operant foraging tasks. Anim Behav 114:189-198. https://doi.org/10. 1016/j.anbehav.2016.02.006

van Horik JO, Langley EJG, Whiteside MA, Laker PR, Beardsworth CE, Madden JR (2018) Do detour tasks provide accurate assays of inhibitory control? Proc R Soc B 285:20180150. https://doi.org/10. 1098/rspb.2018.0150

Vernouillet A, Anderson J, Clary D, Kelly DM (2016) Inhibition in Clark's nutcrackers (Nucifraga columbiana): results of a detourreaching test. Anim Cogn 19:661-665. https://doi.org/10.1007/ s10071-016-0952-y 
Vernouillet AAA, Stiles LR, McCausland AJ, Kelly DM (2018) Individual performance across motoric self-regulation tasks are not correlated for pet dogs. Learn Behav 46:522-536. https://doi.org/10. 3758/s13420-018-0354-x

Veron JEN (1969) An analysis of stomach contents of the water skink, Spenomorphus quoyi. J Herpetol 3:187-189

While GM, Chapple DG, Gardner MG, Uller T, Whiting MJ (2015) Egernia lizards. Curr Biol 25:R593-R595. https://doi.org/10.1016/ j.cub.2015.02.070
Wilkinson A, Huber L (2012) Cold-blooded cognition: reptilian cognitive abilities. In: Vonk J, Shackelford TK (eds) The Oxford Handbook of Comparative Evolutionary Psychology. Oxford University Press, Oxford, pp 129-141

Publisher's note Springer Nature remains neutral with regard to jurisdictional claims in published maps and institutional affiliations. 